Meta

Journal des tradlucteurs

Translators' Journal

\title{
Quel texte traduire ? Le traducteur face au conflit des interprétations
}

\section{Jan de Waard}

Volume 32, numéro 1, mars 1987

La traduction biblique

Bible Translation

URI : https://id.erudit.org/iderudit/004582ar

DOI : https://doi.org/10.7202/004582ar

Aller au sommaire du numéro

Éditeur(s)

Les Presses de l'Université de Montréal

ISSN

0026-0452 (imprimé)

1492-1421 (numérique)

Découvrir la revue

Citer cet article

de Waard, J. (1987). Quel texte traduire ? Le traducteur face au conflit des interprétations. Meta, 32(1), 16-20. https://doi.org/10.7202/004582ar d'utilisation que vous pouvez consulter en ligne.

https://apropos.erudit.org/fr/usagers/politique-dutilisation/ 


\section{QUEL TEXTE TRADUIRE? LE TRADUCTEUR FACE AU CONFLIT DES INTERPRÉTATIONS}

JAN DE WAARD

Institut biblique pontifical, Rome, Italie

Qu'on ne traduit jamais un texte, mais seulement une des multiples interprétations d'un texte, est une conception qui commence à être acceptée même dans les cercles qui s'occupent de la traduction biblique. Quoique ces interprétations soient aussi bien synchroniques que diachroniques, ce sont surtout les dernières qui intéressent le traducteur d'un texte ancien ${ }^{1}$. Car celui-ci, dans sa souvent longue histoire qui va de sa genèse orale jusqu'à sa forme finale écrite, a été l'objet de nombreuses relectures. Même si ces relectures restent entièrement hypothétiques, elles peuvent quand même être postulées avec un degré plus ou moins élevé de probabilité. En outre, le traducteur qui rend un texte biblique dans une langue contemporaine, ne peut faire abstraction de la longue histoire des traductions bibliques antérieures qui présentent elles-mêmes un grand nombre de relectures. La traduction grecque du Psaume 29 (28) telle qu'on la trouve dans la Septante, en est un bel exemple ${ }^{2}$. Tout cela justifie la question fondamentale : quel texte, quelle relecture du texte, et quelle relecture à quelle puissance, faut-il traduire ? Le but de cette contribution est de démontrer ces problèmes dans une unité de discours prophétique, Esaïe 7. 1-17, et d'esquisser quelques solutions possibles ${ }^{3}$.

L'unité de ce discours est indiscutable. Elle se montre, d'une part, par une définition négative des limites du texte, et, d'autre part, par la clarté de la structure interne. Quant à la délimitation négative, il faut bien avouer que la structure de la section précédente, Esaïe 6. 6-13, n'est pas très marquée au début et à la fin. Néanmoins, la présence du mot crochet 'adam (personnes) dans $11 \mathrm{c}$ et $12 \mathrm{a}$ montre les attaches avec ce qui précède, tandis que la répétition du vocable matsebet (souche) sous forme de chiasme en 13 indique la fin d'une section. Par contre, la section qui vient immédiatement après, 7 . 18-25, a une structure hautement formalisée du type A B A C A C. Ces découpages sont déjà des indices précieux, et ils sont confirmés par la structure primaire du discours 7 . 1-17 même. C'est d'après celle-ci que ce texte est partiellement présenté ici, d'abord en hébreu translitéré, puis en traduction française littérale, dans l'arrangement spatial (p. 17).

Cet arrangement spatial est pratiquement identique à celui de $\mathrm{D}$. Winton Thomas dans la Biblia Hebraica Stuttgartensia. Seulement une correction s'impose. Vu le parallélisme structurel entre 7 et $9 \mathrm{~b}$ (deux fois la particule de négation suivie d'un verbe conjugué), la formule d'introduction de 7a (Ainsi a dit le Seigneur Iahvé) ne peut pas faire partie de la structure interne de 7-9b. La double distance de neuf lignes est due au hasard de l'édition et ne doit donc pas être prise à la lettre. Le chiffre indique uniquement que la longueur totale des dialogues antérieurs au discours central est identique à celle des dialogues postérieurs, même si leur nombre est différent. D'ailleurs, le type prédo- 
wayehi bime 'achaz ben yotam ben 5uziyahu melek yehudah

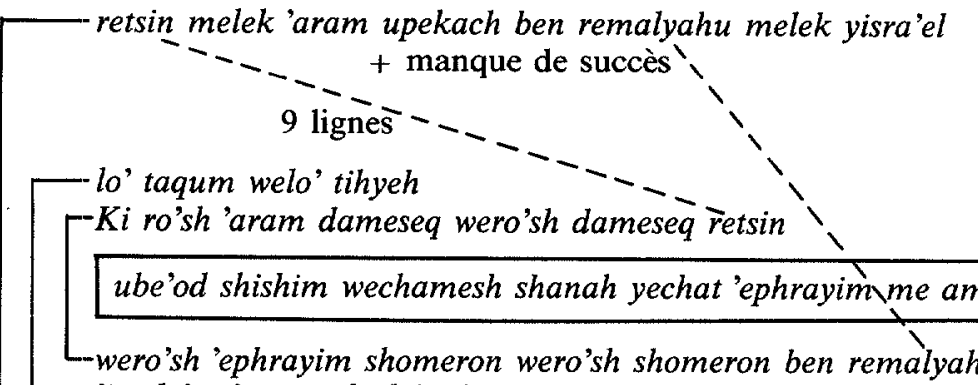

'im lo' ta'aminu ki lo' te'amenu

\section{9 lignes}

melek 'ashur + succès

Et il advint dans les jours d'Achaz, fils de Jotham, fils d'Ozias, roi de Juda

Rason, roi d'Aram et Péqakh, fils de Remalyahou, roi d'Israël

- - + manque de succès

9 lignes

Cela ne tiendra pas

et cela ne sera pas

$\frac{1}{\text { pas }}$

Car la tête d'Aram, c'est Damas, et la tête de Damas c'est Tason 8 a

Dans un délai de 65 ans Ephraïm écrasé ne sera plus un peuple $8 \mathrm{~b}$

\section{$8 b$}

et la tête d'Ephraïm, c'est Samarie et la tête de Samarie c'est le fils de Remalyahou

si vous ne tenez ferme

vous ne serez pas affermis ${ }^{4}$

9 lignes

minant de discours, le dialogue, suggère déjà l'importance des participants à la communication et on peut noter que ceux-ci ont été organisés selon le pattern suivant :

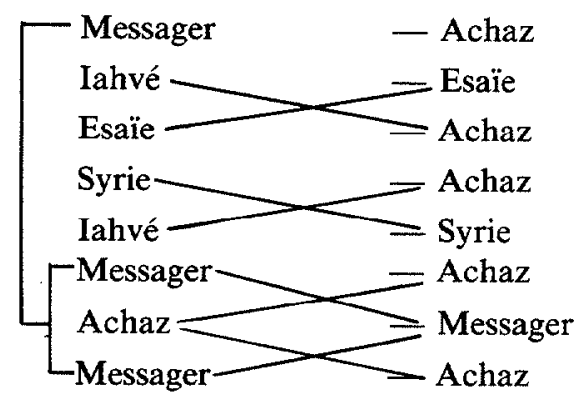


Chaque texte ayant de multiples structures, il serait possible de trouver et de décrire d'autres structures secondaires et tertiaires. Cependant, pour le but de cet article, la description de la structure primaire est largement suffisante.

Il est clair que celle-ci est une structure inversée ${ }^{5}$ qui a son apogée au centre, même s'il y a un autre point culminant dans " l'enveloppe " du verset 17 par la mention mystérieuse du roi d'Assur ${ }^{6}$. Dans le réarrangement du texte, 7 correspond maintenant à $9 \mathrm{~b}$ tandis que $8 \mathrm{a}$ est balancé par $9 \mathrm{a}$. Seul $8 \mathrm{~b}$ est sans parallèle, ce qui crée la structure bien connue de A B C B' A'. C'est la simple méconnaissance de ces structures qui cause même chez des commentateurs récents des affirmations comme la suivante : « (8b) ist sekundärer Einschub, was sich auch daran zeigt, dass die Zeile den Zusammenhang unerträglich unterbricht ${ }^{7}$. Il est évident pour ceux qui ont maintenant 1 'habitude de discerner ces structures, fréquentes dans tous les types de discours de l'Ancien Testament, qu'il n'y a ni " interruption ", ni " interruption intolérable ", mais plutôt mise en valeur d'une certaine information par sa position au centre de la structure. Il n'y a aucune raison donc d'émettre l'autre hypothèse selon laquelle des éléments du texte auraient subi des déplacements accidentels. Pourtant, c'est sur la base d'une telle supposition qu'un certain nombre de traductions, comme la Bible de la Pléiade et la New American Bible, transposent $8 \mathrm{~b}$ après $9 \mathrm{a}$ dans la quasi certitude d'être plus fidèles à un original dont l'existence n'a jamais été attestée. Il n'est peut-être pas inutile de noter que la même transposition se trouve également dans bon nombre de versions qui suivent le principe de l'équivalence fonctionnelle, comme le Français courant et Parola del Signore. Toutefois, dans ce dernier cas, les mobiles d'une telle restructuration ne doivent pas être cherchés dans un original présupposé, mais plutôt dans la langue d'arrivée. À tort ou à raison, on pense que la structure inversée est absente de la langue, ou bien qu'elle existe, mais manque de clarté vis-à-vis des récepteurs peu avertis. Cette autre motivation de la même transposition est normalement reconnaissable à un plus haut degré d'explicitation des relations entre phrases, comme en témoigne la traduction italienne :

Com'è vero che Damasco è la capitale di Aram et Rezin è il capo di Damasco, come è vero che Samaria è la capitale di Efraim e Pekach è il capo di Samaria, fra sessantacinque anni non ci sarà più̀ il popolo di Efraim.

Seule une restructuration translationnelle qui se base sur des critères de la langue réceptrice peut être justifiée.

S'il est relativement clair qu'il faut rejeter une traduction qui s'appuie sur un original reconstitué, il est beaucoup moins simple de définir la façon dont le traducteur doit procéder dans le cas d'une relecture du texte. Tout d'abord, il faut essayer de constater s'il y a relecture ou non, ce qui n'est pas chose facile. Dans 8 b déjà le chiffre " soixantecinq " est assez curieux par sa précision unique. Maintenant, si l'on compte soixantecinq ans à partir de la guerre syro-éphraïmite de 733, on n'arrive pas à la chute de Samarie, capitale d'Efraïm, car celle-ci a eu lieu seulement une dizaine d'années plus tard, en 721. Cette "faute de calcul " pourrait être considérée comme une preuve de l'intégralité de l'original et de l'absence d'une relecture. Car il est sûrement vrai que dans le cas d'un soi-disant vaticinium ex eventu on aurait rencontré un calcul exact! Un tel raisonnement rendrait également superflue l'explication tendant à montrer pourquoi plus tardi- 
vement une glose a été insérée précisément à l'endroit occupé par $8 \mathrm{~b}$. Il est vrai qu'il n'y a qu'une seule explication : étant donné la fréquence de deux structures différentes : A B B' A' et A B C B' A' (ou : A B OB' A' et A B C B' A'), le glossateur change de type de structure en produisant l'information qui entre (et qui ne peut entrer que) dans la position C.

Cependant, cette explication obligatoire est lourde de conséquences. Car elle implique que de telles structures n'étaient pas du tout employées d'une manière inconsciente, comme on l'a parfois suggéré, mais qu'elles faisaient, par contre, partie d'une technique rhétorique consciente. En plus, vu l'intention certaine de l'auteur, glossateur, rédacteur, etc., de communiquer l'information de ce texte à un public étendu, il faut aussi supposer que les lecteurs, et même les auditeurs, de ce texte étaient capables de comprendre la signification d'une structure primaire. Autrement dit, qu'ils étaient donc prédisposés à détecter la présence ou l'absence d'un message dans la position C. Voici les conséquences inévitables que pourtant personne n'a tirées jusqu'à présent ou que personne n'a voulu tirer. Dans l'absence inquiétante de toutes ces considérations, la critique est presque unanime à prétendre quand même qu'il s'agit dans le cas de $8 \mathrm{~b}$ d'une glose plus tardive ${ }^{8}$. Un calcul précis à partir de la guerre syro-ephraïmite mène jusqu'à la fin du règne d'Assarhaddon, roi d'Assyrie, et jusqu'au début du règne de son successeur Assourbanipal. Et Esdras 4.10 parle "des autres populations déportées par le grand et illustre Asnappar (= Assourbanipal), installées dans les villes de la Samarie... ". C'est à de telles déportations, probablement dans les deux sens, que la glose $8 \mathrm{~b}$ ferait alors allusion.

Maintenant, que doit faire le traducteur face à ce conflit des interprétations ? Évidemment, il y a la solution de facilité qui consiste à nier simplement l'existence de tout problème en prenant comme texte source le texte dans sa rédaction finale. Par rapport à la traduction de textes religieux, on constate souvent ce consensus tacite. Sans contester le caractère raisonnable d'un tel consensus, il faut quand même admettre qu'il a souvent été inspiré par une position purement doctrinale, à savoir la forme "canonique» du texte. Il faut aussi avouer que le traducteur consciencieux, même s'il partage ce consensus, ne peut pas l'appliquer toujours et partout. Autrement dit, la question reste entière. Quant au traducteur donc, qu'il adopte l'hypothèse de la glose ou de la relecture, - et comment pourrait-il réagir autrement face au consensus des commentateurs ? - comment doit-il procéder ? Est-ce qu'il doit suivre l'exemple de Moffatt, d'Alexander R. Gordon et de la Revised Standard Version, et mettre avec eux $8 \mathrm{~b}$ entre parenthèses pour ainsi marquer son caractère secondaire ? La réponse doit être négative. Mieux vaut laisser le centre de la structure vide dans la traduction, mettre la glose en note et en expliquer le pourquoi. Car les parenthèses finissent toujours par être diversement interprétées, et elles mettent en évidence un conflit qui n'a pas été senti comme tel tout au long de l'histoire du texte. On peut difficilement concilier les parenthèses et la mise en valeur particulière de 8 b d'après la relecture telle qu'elle fonctionne encore au niveau de la rédaction finale. Ou bien - deuxième option, peut-être préférable - le traducteur peut se laisser guider par l'optique de la relecture qui coïncide avec la rédaction finale, en formulant dans la langue d'arrivée l'équivalent fonctionnel de la mise en valeur occasionnée par la structure dans la langue source. Il n'y a qu'une traduction - allemande celleci (Gute Nachricht) - qui a utilisé ce moyen en rendant 8 b de la façon suivante : $U n d$ das Reich der Leute von Efraim? in 65 Jahren wird es am Ende sein, und das Volk wird nicht mehr bestehen. "Seulement, _ et contrairement à ce qui s'est passé dans cette dernière traduction - une note, dans un langage adapté aux récepteurs envisagés, doit expliquer qu'il s'agit ici d'une nouvelle accentuation du texte due à une relecture. 
Ainsi, un comportement a été esquissé et suggéré qui, par extrapolation, va loin au-delà des limites du texte-exemple. Et, en ce qui concerne la fonction rhétorique des structures, des questions ont été soulevées qui nécessitent des recherches plus amples. De toute manière, on ne peut nier, ni dans l'analyse de l'original ni dans sa traduction, la réinterprétation vivante. C'est elle qui caractérise la véritable tradition.

\section{Notes}

1. Pour les problèmes de synchronie et de diachronie voir surtout le chapitre sur "Structure et herméneutique ", dans Paul Riccur (1969) : le Conflit des interprétations, Paris, Éditions du Seuil, pp. $31-63$.

2. Voir surtout N.A. van Uchelen (1969-70) : De LXX-interpretatie van Ps 29, Nederlands Theologisch Tijdschrift 24, pp. 171-181.

3. L'analyse qui suit faisait partie d'une analyse structurelle plus ample de Esaie $6.1-96$. Celle-ci a été présentée lors d'une rencontre de l'European Regional Translation Committee, le 18 septembre 1985 à Copenhague, en même temps que des observations exégétiques et translationnelles préparées par H.P. Rüger et R. Kassühlke. L'auteur tient à exprimer sa reconnaissance à ces deux chercheurs pour leurs observations pertinentes.

4. Comme le texte hébreu l'a déjà montré, il s'agit d'un jeu de mots que le traducteur doit s'efforcer de rendre d'une façon naturelle. La traduction de Luther dans la Bible allemande de 1545 a souvent été citée comme exemple d'une traduction réussie : "Glaubt ihr nicht, so bleibt ihr nicht", et elle a encore inspiré la traduction allemande récente de la Gute Nachricht : "Vertraut auf den Herrn! Wenn ihr nicht bei ihm bleibt, dann bleibt ihr überhaupt nicht ". D'autres équivalences fonctionnelles se trouvent dans le Français courant : "Vous ne pourrez tenir bon qu'en vous tenant au Seigneur " et dans la Today's English Version : "If your faith is not enduring, you will not endure". Au sujet des jeux de mots, voir $\mathbf{J}$. de Waard, "What about the Fun of the Pun? Word-plays and their Translation ", in : S. Meurer, Ed. (1983) : Mittelpunkt Bibel, Stuttgart, Deutsche Bibelgesellschaft, pp. 79-88.

5. Voir surtout N.W. Lund (1942) : Chiasmus in the New Testament, Chapel Hill, University of North Carolina Press, et John W. Welch (Ed.) (1981) : Chiasmus in Antiquity, Hildesheim, Gerstenberg Verlag. La structure inversée de Es 7. 7-9 a été bien relevée par H. Kosmala (1964: "Form and Structure in Ancient Hebrew Poetry ", Vetus Testamentum 14, pp. 423-445). Parlant de ces versets il remarque : It has an introductory line of four and a concluding line also of four units; they correspond to each other. Between these are three lines of seven units each; the middle line forms the axis and contains the important prophecy" (443-4).

6. Les relations entre "enveloppe" et centre ont été bien discernées par H. Wildberger (1969) : Jesaja. Biblischer Kammentar, Neukirchen, Neukirchener Verlag, p. 298.

7. Ibidem, p. 266.

8. Â partir de G.B. Gray (1912) : The Book of Isaiah, Edinburgh, T \& T Clark, jusqu'à R.E. Clements (1980) : Isaiah 1-39, London, Marshall, Morgan \& Scott. 